\title{
Sentidos sobre as línguas em contextos multilíngues a partir do discurso político-educacional ${ }^{1}$
}

\author{
Sentidos sobre las lenguas en contextos multilingües a partir del discurso \\ político educacional
}

\author{
Concepts of language conveyed in multilingual contexts through an \\ analysis of the political-educational discourse
}

Laura Fortes ${ }^{2}$

Liz Leticia Martinez Ramirez ${ }^{3}$

\begin{abstract}
Resumo
Este artigo apresenta os resultados de pesquisa realizada como parte de uma etapa do projeto Sentidos de línguas em contextos multilingues do discurso político e educacional. Como parte da metodologia escolhida, constituiuse um arquivo que inclui leis e instrumentos normativos cujos conteúdos são relevantes tanto em termos de educação, quanto em termos político-linguísticos. Os documentos foram selecionados e analisados discursivamente, a fim de compreender os sentidos que constituem as relações entre as línguas no Paraguai, especificamente no campo educacional. Teorias com enfoque em uma abordagem translíngue foram mobilizadas para o estudo de práticas e políticas linguísticas a partir de uma visão crítica às orientações denominadas monolíngues. Discute-se como a construção dos sentidos sobre bilinguismo é instituída oficialmente, como se estabelecem as relações desses sentidos com orientações monolíngues e como estas afetam o âmbito políticoeducacional.
\end{abstract}

Palavras-Chave: Análise de Discurso; Bilinguismo no Paraguai. Multilinguismo.

\section{Resumen}

Este artículo expone las conclusiones de las investigaciones realizadas como parte de una etapa del proyecto Sentidos sobre las lenguas en contextos multilingües a partir del discurso político-educacional. Como parte de la metodología escogida se constituyó un archivo que incluye leyes e instrumentos normativos cuyos contenidos son relevantes en cuanto a lo educativo como también a lo político-lingüístico. Los documentos fueron seleccionados y analizados discursivamente con la finalidad de comprender los sentidos que constituyen las relaciones entre las lenguas en Paraguay, específicamente en el ámbito educativo. Se han tomado teorías referentes al abordaje translingüe para el estudio de las prácticas y políticas lingüísticas desde una visión crítica a orientaciones denominadas monolingües. En este artículo se analiza cómo es la construcción de los sentidos

\footnotetext{
${ }^{1}$ Artigo apresentado no Simpósio Temático 01 - A perspectiva intercultural em estudos sobre língua(s) de imigrantes e de fronteiras, durante o II Seminário Latino-Americano de Estudos em Cultura - SEMLACult em Foz do Iguaçu/PR, Brasil, 2018.

${ }^{2}$ Doutora em Letras; Universidade Federal da Integração Latino-Americana (UNILA); Membro do Grupo de Estudos e Pesquisa em Língua Estrangeira, Discurso e Identidade (LEDI-USP); Membro do Grupo de Pesquisa Linguagem, Política e Cidadania (UNILA); Foz do Iguaçu, Paraná, Brasil; laura.fortes@unila.edu.br.

${ }^{3}$ Acadêmica do Curso de Música; Universidade Federal da Integração Latino-Americana - UNILA; Bolsista de Iniciação Científica no Projeto $O$ currículo como instrumento linguístico: ordem e organização de saberes em contextos educacionais multilíngues e translíngues (UNILA-PRPPG-2016-2018); Foz do Iguaçu, Paraná, Brasil; lizlemar04@gmail.com.
} 
sobre el bilingüismo instituido de modo oficial, las relaciones de estos sentidos con orientaciones monolingües y cómo esto afecta al ámbito político-educativo.

Palabras claves: Análisis de Discurso; Bilinguismo en Paraguay; Multilingüismo.

\begin{abstract}
This paper presents conclusions of an ongoing research project, which aims at understanding different concepts of language conveyed in multilingual contexts. Drawing from an analysis of the political-educational discourse, our main objective is to understand the meaning production embedded in the relationship established among languages. Relevant educational laws and documents in Paraguay have been selected and analyzed in order to demonstrate how the construction of bilingualism concepts have been institutionalized and how they affect those meanings produced by the political-educational field. The analysis draws upon Michel Pêcheux's French Discourse Analysis, as well as on a translingual approach to language, which attempts to understand linguistic practices in a more flexible way, building a critical view of monolingual orientations towards language and language teaching practices.
\end{abstract}

Keywords: Bilingualism in Paraguay; Discourse Analysis; Multilingualism.

\title{
1. Introducción
}

Esta investigación constituye una etapa importante del proyecto El currículo como instrumento lingüístico: orden y organización de los saberes en contextos educacionales multilingües $y$ translingües ${ }^{4}$ cuyo principal objetivo ha sido comprender las relaciones discursivas que se constituyen en las diversas prácticas pedagógicas significadas por los currículos de lenguas en la educación básica en contextos educacionales multilingües y translingues.

A partir de una perspectiva teórica discursiva en relación de entremedio con otros campos del saber pertinentes a los estudios del lenguaje, tenemos la intención de de contribuir para la construcción de un archivo de textualidades del discurso político educacional que producen sentidos y saberes sobre las lenguas en distintos contextos. En línea con los objetivos del Proyecto, esta investigación tuvo como principal objetivo teórico-metodológico contribuir para la amplificación de las relaciones de entremedio teórico entre Análisis del Discurso, la Lingüística Aplicada y la Historia de las ideas lingüísticas. Además de eso, se buscó, también, colaborar en las discusiones en torno del currículo, especialmente lo que tiene relación a las situaciones educacionales multilingües y translingües.

Para el análisis discursivo realizamos la configuración de un archivo político educacional que permitió la lectura de los procesos de significación a los cuales nos dedicamos en esta etapa de la investigación. Para eso, fue necesario un levantamiento

\footnotetext{
${ }^{4}$ Proyecto en andamiento UNILA-PRPPG-PIA948-2017. Cf.: Fortes, 2018).
} 
bibliográfico de documentos político educacionales en vigencia referentes a comunidades escolares insertas en contextos bilingües y multilingües.

A partir de la definición de archivo como un "campo de documentos pertinentes o disponibles sobre una cuestión (PÊCHEUX [1982] 2010, p. 51), consideramos el levantamiento de ese material de análisis como un gesto de configuración y lectura del archivo, lo que implica necesariamente trabajar la relación entre lengua y discursividad, o como explica Pêcheux ([1982] 2010, p. 58), "la relación entre la lengua como sistema sintáctico intrínsecamente pasivo de juego, y la discursividad como inscripción de efectos lingüísticos materiales en la historia”. En esa perspectiva, procedemos al análisis discursivo del archivo político-educacional construido en esa etapa de la investigación.

Para la construcción del archivo político educacional fueron seleccionadas las leyes paraguayas consideradas más pertinentes con respecto al tema a ser estudiado. Tomamos como base la Constitución Nacional, promulgada en 1992 y vigente hasta hoy, la Ley de lenguas $N^{\circ} 4251 / 10$ y Ley General de Educación $N^{\circ} 1264 / 98$. A través de una petición en el sitio web de transparencia nacional del gobierno paraguayo, pudimos hacer una solicitación al ministerio de educación de los datos sobre el marco regulatorio sobre las lenguas en el ámbito educativo, gracias a eso pudimos trabajar con datos gubernamentales oficiales. Por medio de una búsqueda en el sitio web del ministerio de educación accedimos a los planes curriculares de la educación media publicados en el portal. A partir de estos documentos se hizo un recorte de los ejemplos textuales tomados para el análisis.

\section{Reflexiones teóricas: sentidos de lengua en contextos multilingües/translingües}

\subsection{Naturalización del monolingüismo}

Ante la cada vez más desafiante realidad lingüística en los espacios educativos surgen profundos cuestionamientos sobre la enseñanza de lenguas en contextos multilingües/translingües. Una de las primeras ideas en cuestionamiento es la naturalización del monolingüismo, sobre la cual se ha construido una forma de pensamiento estática de las lenguas. El monolingüismo es tratado como la norma en cuanto al uso de lenguas, cuando lo más común es la existencia de comunidades que son, por lo menos, bilingües. Siendo el multilingüismo la norma, el monolingüismo debería ser considerado como desvío de la misma (CAVALCANTI, 1999).

Rocha y Maciel (2015) expresan que el monolingüismo es ilusorio y toman como parámetro más realista una orientación translingüe. El término "translingüismo" intenta 
corresponderse en mayor medida con la realidad lingüística. Así el uso del término "translingüe" es, en palabras de los autores, una forma de "resistencia", ya que abraza las diversas, heterogéneas y complejas relaciones que surgen a partir de la realidad lingüística que es sumamente plural. A partir del abordaje translingüe se pretende pensar en los espacios multilingües desde una visión que permita acoger de forma positiva la diversidad y pluralidad cultural.

Entendemos que el impacto de la orientación monolingüe, no se restringe solo a un ámbito teórico, a una puja de ideas sobre las lenguas, sino que refuerza relaciones de dominación/colonización, a través del lenguaje. Se torna importante analizar si los instrumentos políticos toman en cuenta reflexiones de este tipo o simplemente favorecen a la mercantilización de las lenguas, pues entendemos como Rocha y Maciel (2015) que es necesario pensar en cómo la forma en que se dan las prácticas lingüísticas impacta en la realidad, ocuparse de las "formas en que los recursos lingüísticos se ponen en circulación en nuestras sociedades y de qué modo la circulación de estos recursos reproduce, negocia y cuestiona la diferencia y la desigualdad social" (ROCHA y MACIEL, 2015, traducción nuestra).

\subsection{Realidad y contexto educativo}

La predominancia de orientaciones monolingües en el ámbito educativo implica una invisibilización de la diversidad cultural y lingüística. En un relevamiento interesante Cavalcanti (1999) categoriza diversos procesos a través de los cuales en Brasil se generan comunidades/espacios de diversidad lingüística asociada a una heterogeneidad cultural gestada históricamente. Entre esos contextos categorizados podemos citar: indígena, de inmigración, de frontera, comunidad de sordos y lo que la autora llamará como contextos bidialetales, introduciendo un término que explica muy bien realidades lingüísticas comunes en nuestra sociedad.

Hablar de contextos bidialetales es referirse a poblaciones que hablan una variedad del idioma tenido como padrón, que presenta diferencias con esa lengua padrón y que generalmente es tenida como inferior; esta noción de inferioridad es atravesada por cuestiones culturales y sociales. La idea de Rocha y Maciel (2015) de que comúnmente las personas que son tenidas como monolingües, en la práctica social, transitan en realidad en medio de una diversidad de "códigos, registros y discursos" es compatible con la caracterización de los contextos bidialetales realizada por Cavalcanti (1999), cuya importancia reside en entender 
cómo se constituyen nuestras sociedades lingüística y culturalmente, ya que algunos de estos contextos son comunes en los países latinoamericanos. Podemos tomar como nuestra la preocupación de la autora por tornar visibles estas mayorías tratadas como minorías y, más aún, en relación a los espacios educativos.

La visibilidad de los diversos contextos es importante en la educación, y, reflexionando al respecto, Rocha y Maciel (2015, p. 432) harán énfasis en que una pedagogía que "comparte las premisas de una orientación translingüe buscará la comprensión de los paisajes lingüísticos que representan nuestra realidad y la realidad en nuestras salas de clase, en medio de toda su heterogeneidad". Por último podemos tomar una observación interesante de los autores, que, apoyados en Canagarajah, plantean:

Como advierte Canagarajah (2013, p. 191), las prácticas educativas translingües no implican, necesaria y exclusivamente, el rompimiento con "normas todo el tiempo". La orientación translingüe, en el campo educativo, incide, principalmente, en desarrollar una postura crítica frente al modo como las normas se constituyen, para que puedan ser desarrolladas, a lo largo del proceso educativo, capacidades, estrategias y condiciones de negociación a partir de la y en medio a la diversidad. El fortalecimiento de esa postura crítica y performativa, según el autor, permite que nos comprometamos creativamente en situaciones de uso del lenguaje, con base en contextos específicos y objetivos particulares, sin suponer homogeneidad. (ROCHA; MACIEL, 2015, p. 434, traducción nuestra).

Esto significa que no escapamos a los patrones que normatizan el uso de la lengua, pero existen momentos en los que ellos pueden ser quebrados, a través de una "postura crítica" que permite una actuación consciente a la hora de abordar estrategias que permitan generar "condiciones de negociación" en contextos de diversidad.

\subsection{Políticas regionales}

Rocha y Maciel (2015), al pensar la cuestión pedagógica de la lengua, toman un concepto importante para entender la naturaleza de los espacios en los que nos encontramos con contextos en donde la diversidad es predominante, así los autores aplican el término espacio aporético y lo explican de la siguiente manera: "podemos delinear nuestra concepción de espacios aporéticos como aquellos en que, a partir de la duda, de la incertidumbre, de la inestabilidad y del conflicto, posibilitan desplazamientos, llevándonos a construir otros sentidos" (ROCHA; MACIEL, 2015, p. 436, traducción nuestra).

Dar un nombre que nos permita entender la inestabilidad y la situación de frontera cultural es relevante a la hora de comprender la realidad, así cuando hablamos de espacios aporéticos esto nos trae en mente los desafíos que estos lugares representan, la necesidad de 
replantearnos la forma de entender estos contextos y "rediseñar nuestras ideas, dichos y haceres" como dirán Rocha y Maciel (2015, p. 436), que muchas veces seguirán siendo dispares. Aceptar la disparidad como parte de los espacios es algo muy positivo que se presenta con ese término.

Al tocar la cuestión de la pedagogía pública, los autores anteriormente citados también hacen una distinción entre espacio público y privado. Tomaremos aquí la definición que nos interesa, la de espacio público. El espacio público no se limita a un espacio físico, debe ser pensado teniendo en cuenta el bienestar del otro en medio de las relaciones sociales, lo que conlleva entender el mundo de los otros, las otras realidades, la heterogeneidad del modo de pensar y de actuar. Entender la convivencia en medio de una diversidad lingüística y cultural como positiva, asumir la existencia de estos espacios aporéticos que deben ser pensados también como públicos, es decir desde la búsqueda del bienestar del otro, debe ser un punto de reflexión e inflexión a la hora de elaborar políticas regionales.

Entendemos que para repensar conceptos, prácticas pedagógicas, modos de actuar, es necesario entender cómo se constituyen las políticas regionales. Es importante plantearnos si las políticas regionales sobre lenguas que están siendo aplicadas nos ayudan a la flexibilidad en los espacios en donde acontecen las relaciones entre lenguas o si por el contrario constituyen una fuerza que coarta la fluidez, la libertad y la singularidad de cada expresión cultural.

A partir de las consideraciones teóricas presentadas, desenvolvemos un análisis discursivo de los sentidos sobre las lenguas en contextos multilingües a partir de un archivo político-educacional, constituido por diversos documentos e instrumentos estatales.

\section{Relevancia de la lengua oficial en el orden político y educacional}

El sintagma "lengua oficial" es utilizado en diversos países para designar a la lengua instituida como instrumento político-normativo de uso común en las instituciones estatales y sus órganos competentes, ámbito en el que se encuentra el sistema educativo. Como instrumento normativo otorga validez formal a los actos jurídicos desarrollados dentro del territorio nacional. Como instrumento político refuerza la idea de unidad entre nación-estadolengua. Esta relación se expresa más simplemente en la idea de que un país tiene una o más lenguas oficiales, utilizadas dentro de su territorio con preferencia y protección estatal, formando parte de una identidad cultural común. La noción de lengua oficial está fuertemente relacionada a la de lengua nacional. El uso de una lengua oficial no se resume a una función 
instrumental y práctica, tiene la función unificadora del lenguaje dentro de un territorio delimitado y esta unidad está justificada por la noción de identidad nacional.

Los idiomas oficiales del Paraguay son el español y el guaraní según lo establecido en la Constitución Nacional del Paraguay. Este es el instrumento normativo más relevante del país, con vigencia plena desde 1992, año en el que se introdujo por primera vez el idioma guaraní dentro de esta categoría. En la constitución anterior, la de 1967 (PARAGUAY, 1967), el guaraní y el español fueron considerados idiomas nacionales, aunque solo el español era oficial.

Ambos idiomas, reconocidos primeramente como lenguas nacionales en 1967, están caracterizados por una relación construida a lo largo de los años de forma diglósica (MELIA, ([1973] 1988). Meliá ([1973] 1988, p. 113) analizó la situación lingüística en el Paraguay, denominando a la misma como "di-lingüismo", siendo esta resultante del proceso colonial. Al mismo tiempo, según el investigador, se instauró el "a-lingüismo", o sea, un "complejo de inferioridad lingüística" marcado por sentidos de un "no saber hablar" construidos en torno a la lengua guaraní. En contraposición, Verón (2017) resalta:

Tener un $80 \%$ de la población que habla guaraní es la prueba elocuente de la vitalidad de esta lengua, pero es el momento de romper con la diglosia, de elevar el estatus de esta lengua, de modernizarla, de convertirla en un idioma de uso escrito y normal en el Estado y la sociedad. (VERÓN, 2017, p. 118).

Pero el carácter de lengua "nacional" expresa que por lo menos una gran parte de la población tiene al guaraní como lengua materna, y más profundamente se relaciona con construcciones entorno a la formación del concepto de identidad nacional. Esta relación queda más clara cuando en el artículo ${ }^{\circ} 3$ de la Ley de Lenguas $\mathrm{N}^{\circ} 4251$, instrumento normativo promulgado en 2011, se expresa:

Art. $3^{\circ}$.-De las lenguas oficiales. Las lenguas oficiales de la República tendrán vigencia y uso en los tres Poderes del Estado y en todas las instituciones públicas. El idioma guaraní deberá ser objeto de especial atención por parte del Estado, como signo de la identidad cultural de la nación, instrumento de cohesión nacional y medio de comunicación de la mayoría de la población paraguaya. (Destaques nuestros).

La conceptualización de lengua oficial en la primera parte tiene en sí la caracterización de lo oficial. La segunda parte destacada actúa sobreponiendo el atributo de lo nacional sobre el guaraní específicamente. Así queda expresada esta relación ficcional que realiza la Ley de Lenguas, siendo analizada aquí como parte del discurso estatal sobre lenguas, en el cual lo nacional es unido a lo oficial para convertirse en fundamento de la importancia del guaraní (NIRO, 2010) y esta unidad da base a la institución del bilingüismo. 
Es importante señalar que en la Constitución Nacional de 1992, vigente actualmente, se instaura el deber de uso y aceptación del guaraní en las instituciones de gobierno, y también su enseñanza dentro del sistema de educación formal, con esto la política lingüística y el discurso estatal fueron repensados, aunque no necesariamente la reformulación tenga bases la preocupación por la pluriculturalidad, o el derecho a la lengua (BAREIRO SAGUIER, 1990), como el discurso político educacional intenta expresar.

Rubén Bareiro Saguier, quien fuera constituyente en la elaboración de la Constitución Nacional de 1992, habla del derecho a la lengua, como defensa de la inclusión del guaraní en el sistema formal y la educación antes de la promulgación del instrumento normativo que tendría como consecuencia la posterior reforma educativa a través de la cual se incluye el guaraní de manera oficial en el sistema educativo formal. En un fragmento de su defensa el mismo expresa:

Como no se alfabetiza en guaraní, no existe sino una literatura embrionaria en ese idioma. La misma está reducida a una oralidad precaria: letras de canciones, un teatro bastante reprimido y otras manifestaciones más o menos marginales. Nosotros, los autores paraguayos, somos escritores colonizados que hemos interiorizado la condición de tales. No escribimos en guaraní, aunque existe un alfabeto desde el siglo XVI, porque se ha provocado en nosotros la amnesia cultural, desde la infancia, en lo que concierne a la escritura en lengua aborigen. Esta lengua que, sin embargo, hablamos corrientemente en la vida cotidiana. [...] En nombre de esa situación tan generalizada como aberrante vengo, en esta reunión dedicada a «la afirmación, la adaptación y a la extensión de los Derechos Humanos», a reclamar la incorporación prioritaria, en la Declaración de los nuevos derechos humanos, de ese derecho inmemorial, el derecho inalienable a la lengua, el derecho esencial a ser alfabetizado en el propio idioma, en la lengua materna. (BAREIRO SAGUIER, 1990).

En este argumento a favor de incluir al guaraní como idioma oficial, la idea fundamental gira en torno a la alfabetización en guaraní. Este discurso representa una visión que entiende como inferior una lengua de tradición oral. El derecho a ser educado en lengua materna es pensado a los efectos de la alfabetización del guaraní hablante, no como un derecho universal para la educación. La oralidad es el estigma que se busca eliminar, por estar, en esta concepción, relacionada con el atraso y con una "amnesia cultural", amnesia ligada al olvido de las raíces identitarias, por lo que también podemos entender que responde al rescate de lo que identifica al paraguayo.

\section{Bilingüismo en Paraguay: Análisis de los espacios de las lenguas en la educación}


A partir de 1992, con la instauración del guaraní como lengua oficial, la coexistencia de dos lenguas oficiales en Paraguay, el guaraní y el español. El artículo 77 de la Constitución nacional del Paraguay expresa:

La enseñanza en los comienzos del proceso escolar se realizará en la lengua oficial materna del educando. Se instruirá asimismo en el conocimiento y en el empleo de ambos idiomas oficiales de la República. En el caso de las minorías étnicas cuya lengua materna no sea el guaraní, se podrá elegir uno de los dos idiomas oficiales.

Partiendo de este artículo intentaremos entender algunos aspectos de la relación entre las lenguas oficiales/nacionales, el discurso estatal y la relación con otras lenguas en el ámbito educativo.

\subsection{Educación en lengua materna: ¿Expresión o derecho?}

El artículo $\mathrm{N}^{\circ} 77$ de la constitución nacional busca reglamentar la enseñanza en lengua materna y hace mención a introducción expresa del guaraní en la educación formal desde el comienzo del proceso escolar. En la parte final del artículo, dice lo siguiente: "En el caso de las minorías étnicas cuya lengua materna no sea el guaraní, se podrá elegir uno de los dos idiomas oficiales."

Como ya expresamos, el estado reconoce que el bilingüismo es importante, e intenta promover el uso del guaraní y el español en forma igualitaria. Siendo así, diversos instrumentos normativos se ocuparán de intervenir en la educación con la intención de que esta "educación bilingüe" sea efectiva, intentando responder a la necesidad de alfabetización de "guaraní hablantes". Sin embargo, los hablantes de otras lenguas minoritarias o portadores de otras nacionalidades no tienen el mismo estatus y la misma consideración.

Aunque las expresiones anteriormente utilizadas parecen dar cuenta de una especie de "privilegio" hacia el guaraní, el efecto de esta visión estatal sobre las lenguas refuerza la exclusión del guaraní hablante, pues se normatiza al guaraní como si fuese una lengua homogénea, cuando en realidad existen diversas variedades en el uso del guaraní, variedades que también pueden considerarse como excluidas del sistema educativo.

En todos los documentos político-educacionales surgidos a partir de la constitución nacional de 1992, serán tratadas diferencialmente las lenguas oficiales con respecto a otras lenguas como las indígenas o las de migrantes. Las "otras lenguas" aparecerán en artículos diferenciados o con frases que dejan clara esta distinción. En esta diferenciación de espacios textuales, la lengua materna estará en muchos de los casos asociada a la lengua oficial/nacional, ella no es pensada como declaración de un derecho universal, será enunciada 
como expresión testimonial, siempre subyugada a los caracteres de "nacional y oficial". Estas ideas que atraviesan los documentos políticos educacionales generan un contrasentido profundo con el discurso que dará fundamento al bilingüismo, ya que la expresión estatal en defensa del bilingüismo se basa principalmente en el derecho a la alfabetización en la lengua materna, y en valores como el respeto a la pluralidad y los beneficios de la interculturalidad.

La forma de entender el bilingüismo, manifestada en los documentos estatales, es comparable a lo que Garcia y Yip (2017) apuntan sobre cómo existiendo un discurso, que en apariencia puede ser abierto y positivo por parte de autoridades o entes gubernamentales, se pueden promover acciones que responden a una forma restricta de entender las lenguas. Esto acontece cuando se da un espacio selectivo para algunas de las lenguas, en contextos educativos y esto cercena o restringe posibilidades a quienes se encuentren en otros grupos lingüísticos diversos, que no son padronizados.

\section{2 "Lengua oficial materna": entre la necesidad de control estatal y la realidad lingüística}

Queda claro que existen contradicciones en las relaciones entre las prácticas lingüísticas y la forma en que el estado intenta controlar estas relaciones, y estas aparecen en el discurso político educacional sobre las lenguas en forma de equívocos de la textualidad. El equívoco funciona en el discurso como un punto que refleja la incapacidad del lenguaje de ser un sistema absolutamente completo y cerrado. Así, discursivamente, el equívoco nos muestra la posibilidad de dislocar los sentidos, porque el lenguaje es esencialmente susceptible de ser interpretado. "El equívoco irrumpe como un lugar de resistencia que es inherente a la lengua ya su constitución y compatible con la naturaleza inestable, heterogénea y contradictoria de un sistema no cerrado" (FERREIRA, 2000, p. 15, traducción nuestra). Esto es parte del propio funcionamiento de la lengua en la historia, siendo capaz de producir enunciados que pueden tornarse otros en cualquier momento.

El sintagma "lengua oficial materna" aparece en varios documentos, y aquí lo que analizaremos brevemente como un espacio de equívoco. En esta expresión está contenida por un lado la lengua materna y por otro lado la de lengua oficial. La primera hace referencia a aquella lengua aprendida de forma natural por la persona en sus primeros años de vida, tiene que ver con la identidad individual y la segunda es establecida por el estado con la ficción que nace de las formas textuales de las leyes, que demarca su uso oficial y obligatorio en el 
territorio nacional, con lo que se que presupone que son lenguas conocidas y utilizadas por todas las personas.

En las normas, ambos términos son sobrepuestos y, con una mirada atenta, podemos ver como conceptos que parecen tener sentido guardan una profunda contradicción. A través de la normatización se homogeniza la idea sobre la realidad y se naturaliza el término, finalmente la contradicción perdura, tanto en el término como en la realidad, la homogeneidad sólo se da en el discurso. Guimarães (2005) ejemplifica una sobreposición similar existente entre la noción de lengua oficial y lengua nacional y materna en Brasil:

\begin{abstract}
El conjunto de textos que componen este Núcleo Temático muestra cómo el portugués, como lengua materna de Brasil no es necesariamente la lengua materna de todos los brasileños, aunque es siempre, para toda la lengua nacional y oficial de Brasil, incluso a aquellos que no la hablan. Por otro lado, está en relación con un gran número de lenguas de modos bastante diferentes. La característica clave de este conjunto de relaciones es que el portugués como lengua oficial y nacional, y mientras imaginario de unidad, superpone su carácter de lengua oficial y nacional al carácter de lengua materna, y se distribuye a sus hablantes como políticamente dominante. Esto hace que la distribución de las otras lenguas a sus hablantes sea significada por un carácter de "inferioridad". (GUIMARÃES 2005, p. 22)
\end{abstract}

Mientras los instrumentos normativos reconocen que la educación de los niños en su lengua materna es fundamental, a la vez hay una fuerte expresión de imposibilidad de salir de las lenguas oficiales, al punto de dejar constancia de contra sentido en el discurso textual.

La lengua oficial no siempre será materna, y la lengua materna puede no ser la oficial (GUIMARÃES, 2005). Cuando aparece el sintagma "lengua oficial materna" claramente hace referencia a las lenguas oficiales, español y guaraní. De hecho podría expresarse el sentido de los textos normativos en esos términos, por ello la palabra "materna" parece ser necesaria para dar la apariencia ser más considerada con las necesidades y la realidad lingüística del niño. La existencia del adjetivo "materna" es meramente textual, por lo que el derecho a la lengua materna queda subyugado al carácter de la oficialidad.

\title{
3.3 Pluriculturalidad: conflicto con el tratamiento de lenguas
}

Partiendo del análisis de diferentes documentos, que como el artículo $\mathrm{N}^{\circ} 77$ de la Constitución Nacional concentran sus esfuerzos en las lenguas oficiales y que responden a la noción de identidad nacional, anclada fuertemente en la forma de constitución de los estadosnación, hay indicios para observar que en el discurso estatal se encuentra la visión de que la lengua es un reflejo de la identidad nacional (PAYER, 1999), generando un olvido de otras realidades lingüísticas, que no son consideradas o que son consideradas solo de forma testimonial en el discurso. 
Es importante señalar que esta visión sobre las lenguas no contempla los procesos en las aulas, espacios en los que emergen diversas prácticas lingüísticas que se encuentran fuera de estos idiomas oficiales (GARCIA; YIP, 2017). En la textualidad de los dispositivos normativos, a través de los cuales nos proponemos analizar el discurso político educacional se deja ver una intención de abrazar la interculturalidad y pluralidad, pero también es claro que el control estatal opera en sentido diferente a la realidad lingüística. Podemos ver esto brevemente en dos ejemplos textuales:

\section{Artículo 140 - DE LOS IDIOMAS}

El Paraguay es un país pluricultural y bilingüe. Son idiomas oficiales el castellano y el guaraní. La ley establecerá las modalidades de utilización de uno y otro. (PARAGUAY, 1992).

Promover una actitud positiva de los educandos respecto al plurilingüismo paraguayo y propender a la afirmación y al desarrollo de las dos lenguas oficiales. (PARAGUAY, 2014).

El Paraguay posee una pluralidad de culturas representadas por las diferentes etnias que lo habitan y los grupos de inmigración antigua y reciente que lo pueblan. Las ciencias pedagógicas y la didáctica mostrarán los medios y los modos más eficaces para satisfacer las exigencias de este pluralismo cultural, atendiendo especialmente la situación que plantea el bilingüismo, de modo que la expresión en las dos lenguas oficiales sea un vehículo efectivo para la transformación, la promoción y la dinamización de la cultura. (PARAGUAY, 2014).

Tanto en la Constitución Nacional (PARAGUAY, 1992) como en el Plan Curricular de la Educación Media (PARAGUAY, 2014) la caracterización de la pluriculturalidad del país aparece superpuesta con el bilingüismo, así encontramos un espacio que funciona de modo similar al del sintagma "lengua oficial materna". Veamos por qué.

El sentido de la pluriculturalidad nos trae el imaginario de la diversidad de un país en el que habitan personas con varias culturas, desde los pueblos indígenas, migrantes, las variedades culturales regionales que también presentan características propias que las distinguen, etc. El bilingüismo aquí citado está constituido por los aspectos antes analizados, que hacen referencia a la superposición de la lengua oficial con la lengua nacional, produciendo la ilusión de identidad total entre ambos elementos, que tiende a dar un sentido homogéneo al uso de las lenguas oficiales guaraní/español, y a la vez actúa dando bases a la defensa del bilingüismo como elemento constituyente de la identidad paraguaya.

Es claro que la diversidad de un país pluricultural no es atendida por el bilingüismo, pero aquí nuevamente se crea este sentido de que el bilingüismo responde a la construcción de un país pluricultural. Por esta ilusión del discurso nuevamente ocurre la homogeneización de la visión sobre la realidad, en donde el elemento homogeneizante sigue siendo la constitución 
del sentido nacional. Es notable que, cuando en uno de los textos la palabra plurilingüismo aparece, está relacionada a la nacionalidad, así el plurilingüismo es "paraguayo", haciendo palpable que la noción de lo plural no tiene existencia propia, es siempre lo nacional lo que la delimita. O sea, "la historia lingüística de Paraguay es, en gran medida, la historia de la negación del plurilingüismo" (VERÓN, 2017, p. 110).

\section{Conclusiones}

El análisis de los documentos políticos educacionales del Paraguay, con un archivo constituido por leyes y por plan curricular de la educación media, nos permitió entender parte del discurso estatal sobre las lenguas, dándonos algunas nociones sobre qué ideas orientan el orden y la organización de los saberes en el contexto lingüístico paraguayo.

Pese a que la idea de bilingüismo aparece constantemente en los documentos y la coexistencia de dos idiomas oficiales/nacionales, que poseen una relación desigual y desequilibrada, está presente en la discusiones sobre la cuestión lingüística, a través de este análisis del discurso político estatal podemos concluir que esta discusión es desplazada del espacio de habla del estado, en donde los fundamentos a favor de la promoción del guaraní son basados en el uso y la promoción de la lengua como parte de la construcción de lo nacional. Como efecto de un discurso homogeneizante en torno a las lenguas, se produce la omisión de la heterogeneidad cultural que compone la sociedad paraguaya y con esto también se favorece al olvido de las asimetrías más profundas que forman parte de las relaciones entre lenguas.

En las discusiones teóricas presentadas, un concepto fundamental es el de espacio aporético y público. Los espacios aporéticos son aquellos en los que a partir de la inestabilidad, incerteza, duda y conflicto se posibilitan desplazamientos para la construcción de nuevos sentidos (ROCHA y MACIEL, 2015). Este concepto representa un desafío para la política lingüístico-educativa en Paraguay, pues es necesario dar nombre a esos entremedios en donde acontecen las relaciones entre hablantes y lenguas, la aceptación de la existencia de la heterogeneidad, el reconocimiento en el discurso educativo de las realidades que son heterogéneas y de los intercambios fluidos que salen del control del Estado.

Para la educación, la posibilidad de comprender al otro es más relevante que la acumulación de conocimientos linguísticos, juzgados desde una orientación monolinguistica como ser un buen hablante de cualquier lengua. Por ello, en la educación es particularmente necesario representar los espacios de práctica lingüística heterogénea en el discurso político 
educacional. En el aula se representa y vivencia la diversidad, y esta experiencia, desde una orientación translingüe (ROCHA y MACIEL, 2015), se convierte en un espacio de entendimiento del otro, de aprendizaje y de comprensión de la diversidad y la pluriculturalidad.

En este sentido, algunos puntos que sería pertinente estudiar más adelante podrían ser el relacionamiento de las lenguas indígenas, de las lenguas de migrantes y contextos de frontera con las lenguas oficiales del Paraguay, el espacio que ellas ocupan en el discurso político estatal y que sentidos son constituidos sobre estas lenguas.

\section{Referencias}

BAREIRO-SAGUIER, R. De nuestras lenguas y otros discursos. Asunción: Universidad Católica Nuestra Señora de la Asunción, 1990.

CANAGARAJAH, A. S. Translingual Practice: Global Englishes and Cosmopolitan Relations. New York and Abingdon: Routledge, 2013.

CAVALCANTI, M. C. Estudos sobre educação bilíngue e escolarização em contextos de minorias linguísticas no Brasil. In: DELTA: Documentação de Estudos em Linguística Teórica e Aplicada, São Paulo, v. 15, p. 385-417, 1999.

FERREIRA, M. C. L. Da ambiguidade ao equívoco: a resistência da língua nos limites da sintaxe e do discurso. Porto Alegre: Ed. Universidade/UFGRS, 2000.

FORTES, L. O currículo como instrumento linguístico: ordem e organização de saberes em contextos educacionais multilíngues e translíngues. Projeto de Pesquisa em andamento. UNILA. Foz do Iguaçu, 2018.

GARCIA, O.; YIP, J. Translinguagens: Recomendações para educadores. Iberoamérica Social: Revista-Red de Estudios Sociales IX, pp. 164 - 177, Ene 2018.

GUimarÃES, E. Brasil: País Multilíngue. Ciência e Cultura. São Paulo. Vol. 57. n. 2. p. 22-23. Abril/Jun. 2005.2 Disponível em: <http://cienciaecultura.bvs.br/pdf/cic/v57n2/a14v57n2.pdf> Acesso em: 30 jun. 2015.

MELIÁ, B. [1973] Diglossia en el Paraguay (o la comunicación desequilibrada). In: ORLANDI, E. P. (org.) Política Linguística na América Latina, SP: Pontes, 1988.

NIRO, M. El guaraní como lengua oficial: entre el nacionalismo y la integración regional. In: CELADA, M; FANJUL, A. P.; NOTHSTEIN, S. (coords.) Lenguas en un espacio de integración: acontecimientos, acciones, representaciones. Buenos Aires: Biblos (2010).

PARAGUAY. Constitución de la República de Paraguay. Asunción, 1967.

PARAGUAY. Constitución de la República de Paraguay. Asunción, 1992. 
PARAGUAY. Ley General de Educación no 1264. Asunción, 1998.

PARAGUAY. Ley de Lenguas nº 4251. Asunción, 2011.

PARAGUAY: Ministerio de Educación y Cultura. Actualización Curricular del Bachillerato Científico de la Educación Media. Plan Común: Área Lengua, Literatura y sus Tecnologías. Asunción: MEC, 2014.

PAYER, M. O. Memória da língua: imigração e nacionalidade. Tese de Doutorado, IEL, UNICAMP, 1999.

PÊCHEUX, M. [1982] Ler o arquivo hoje. In: ORLANDI, E. (org.) [et al.] Gestos de leitura: da história no discurso. $3^{\text {a }}$ ed. Campinas, SP: Editora da Unicamp, 2010. p. 49-59.

ROCHA, C. H.; MACIEL, R. F. Ensino de língua estrangeira como prática translíngue: articulações com teorizações bakhtinianas. DELTA: Documentação de Estudos em Linguística Teórica e Aplicada, v. 31, p. 411-445, 2015.

VERÓN, M. A. Paraguay: una nación pluricultural con dos lenguas oficiales. Revista de Llengua i Dret, Journal of Language and Law, núm. 67, 2017, p. 106-128. DOI: $\underline{10.2436 / \text { rld.i67.2017.2948. }}$. 